\title{
Handwritten Character Recognition Using Competitive Neural Trees
}

\author{
Theingi Htike and Yadana Thein
}

\begin{abstract}
Neural network classifier methods and decision trees are widely used in various pattern recognition research areas. Among them, handwritten character recognition still faces some issues in all languages. Myanmar handwritten character recognition based on Competitive Neural Trees $(\mathrm{CNeT})$ is proposed in this paper. CNeT performs hierarchical classification and apply competitive unsupervised learning at node label. The goals of Myanmar handwritten character recognition are to obtain better recognition accuracy rate and robust in geometric character shapes of different writing styles. Three main steps such as preprocessing, shape feature descriptors extraction and recognition are implemented in our experiment. Shape feature descriptors are extracted from preprocessed images which are used in Competitive Neural Trees (CNeT) for recognition. This paper discusses a global search method for the CNeT, which is utilized for training.
\end{abstract}

Index Terms-Myanmar handwritten characters, CNeT, global search method.

\section{INTRODUCTION}

Optical Character Recognition (OCR) is a field of research in pattern recognition, artificial intelligence and machine vision. It refers to the mechanical or electronic translation of images of handwritten, typewritten or printed text into machine-editable text. Handwritten character recognition is comparatively difficult, as different people have different handwriting styles. So, handwritten OCR is still a subject of active research. The domain of handwritten text recognition has two completely different problems of On-line and Off-line character recognition.

On-line character recognition [1] involves the automatic conversion of characters as it is written on a special digitizer, where a sensor picks up the pen-tip movements as well as pen-up/pen-down switching. The off-line character recognition is comparatively difficult, as different people have different handwriting styles and also the characters are extracted from documents of different intensity and background [2].

A review of the character recognition work done on Myanmar languages is excellently reviewed. Paper [3] proposed a system to recognize off -line Myanmar handwriting. They used discrete Hidden Markov Model. In paper [4] proposed Handwritten Myanmar Optical Character Recognition System. They used histogram labeling method for recognition. Recognition accuracy rate $98.18 \%$ was obtained.

Manuscript received February 22, 2013; revised April 25, 2013.

The authors are with University of Computer Studies, Yangon, Myanmar (e-mail: htike83@gmail.com).
Paper [5] proposed an effective recognition approach for Myanmar Handwritten Characters. Hybrid approach use MICR (Myanmar Intelligent Character Recognition) and back-propagation neural network. In Hybrid approach, the features of MICR have been used in back-propagation neural network as input nodes. The back-propagation algorithm has been used to train the feed-forward neural network and adjustment of weights to require the desired output. Using Hybrid approach, over-all recognition accuracy of $95 \%$ was obtained.

In this paper, competitive neural trees are proposed for character recognition. In Myanmar character recognition fields, competitive neural trees had not been applied for recognition. Therefore, competitive neural trees are applied to develop off-line Myanmar handwritten recognition system. It is one of the fast supervised neural networks. Neural trees were introduced for character recognition in an attempt to combine advantages of neural networks and decision trees. The aim of the proposed system is to implement an effective approach which is able to recognize for Myanmar handwritten characters. The style of writing characters is different and they come in various sizes and shapes.

The remainder of the paper is organized as follows: Section II describes Handwritten Myanmar language Nature. Section III explains proposed system design and the various steps involved in the OCR System. Section IV presents competitive neural trees and Section $\mathrm{V}$ discusses about experimental results and Section VI describes the conclusion.

\section{HANDWRITTEN MYANMAR LANGUAGE NATURE}

The interests in Myanmar handwritten characters recognition research have grown over the past few years but practical research is only a few works in research field. Because, the problem of Myanmar characters recognition is more difficult than English languages in respects including the similarity of characters, absence space between each word, etc. So, various character recognitions method not enough complete recognize Myanmar character. They are still in research field, not complete work.

Myanmar language is widely used in many offices such as passport, bank and tax etc. So, it is a very importance to develop the high accuracy character recognition system for Myanmar language. Myanmar Language includes Kachin, Kayar, Kayin, Chin, Myanmar, Rakhine and Shan, etc. Myanmar language contains thirty three consonants and a sample of thirty three consonants handwritten character is described in Fig. 1. 


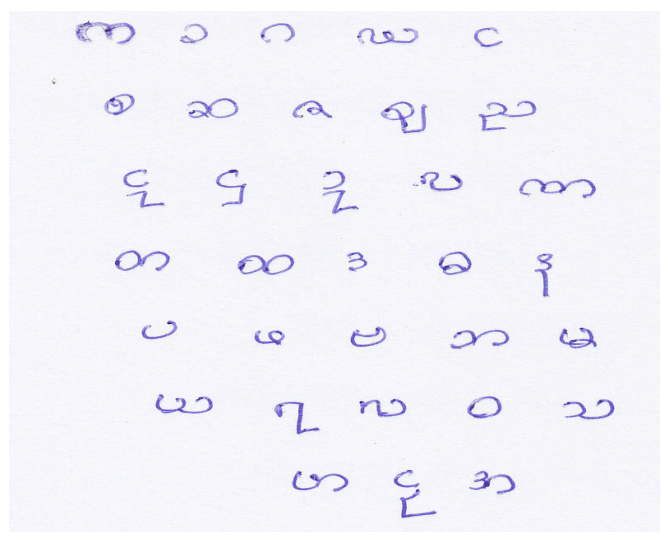

Fig. 1. A set of Myanmar characters

\section{PROPOSED SYSTEM DESIGN}

A typical character recognition system is characterized by a number of steps, which include image acquisition and three core steps as follow:

1) Preprocessing

2) Shape Feature Descriptor Extraction, and

3) Recognition.

The detailed discussion is presented in this section and Fig. 2 shows the step by step procedures.

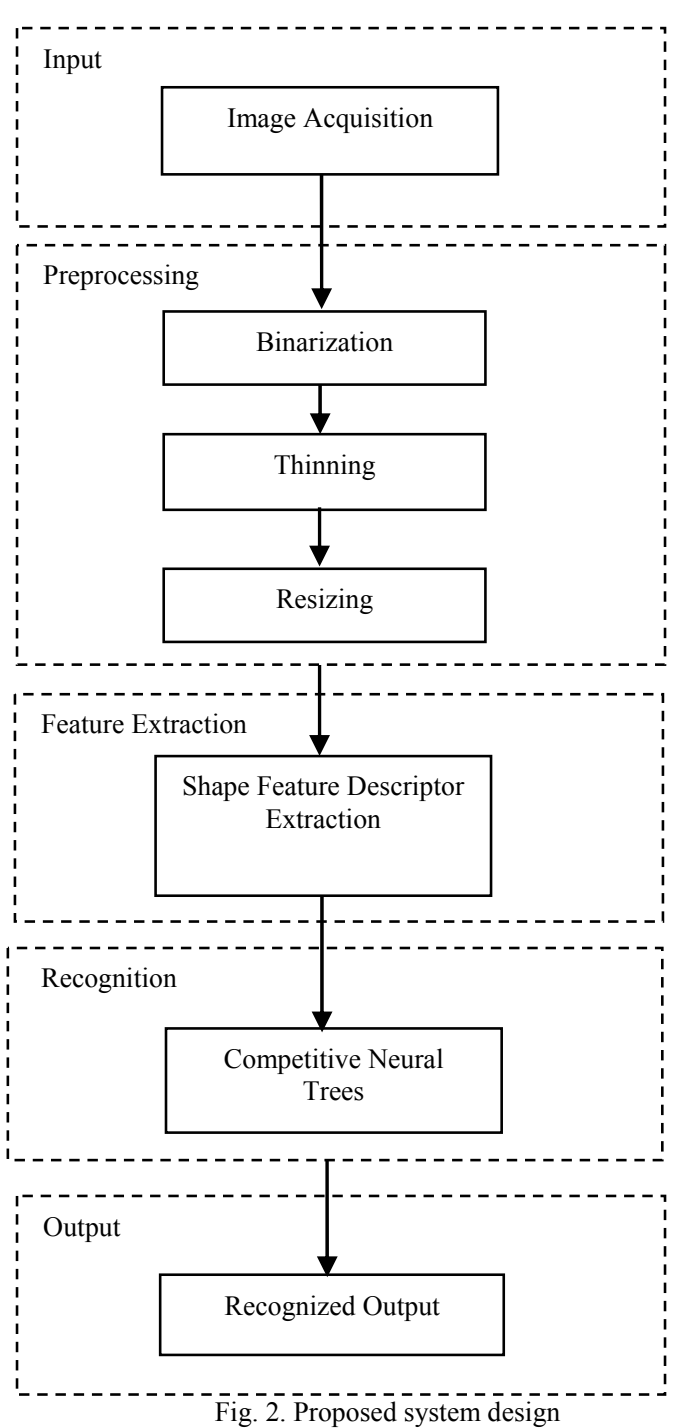

\section{A. Image Acquisition}

It performs getting image from other sources like scanned image.

\section{B. Preprocessing}

Preprocessing aims at eliminating the variability that is inherent in hand-printed characters. The preprocessing techniques that have been employed in an attempt to increase the performance of the recognition process are as follows:

Binarization: All hand printed characters are scanned into gray scale images. Each character image is traced vertically after converting the gray scale image into binary matrix.

Thinning: Thinning is a morphological operation that removes selected foreground pixels from binary images. It is particularly useful for skeletonization. The thinning process reduces the width of pattern to just a single pixel.

Resizing: Each character is resized to extract row and column for each character.

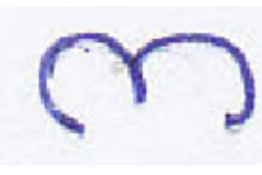

(a) Original Image

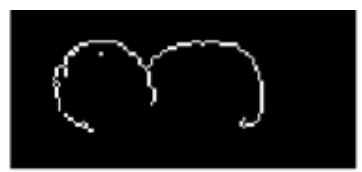

(c)Thin Image

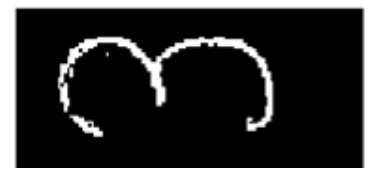

(b) Binary Image

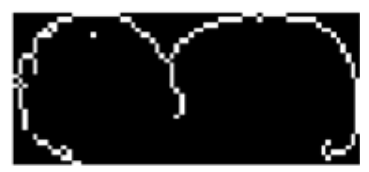

(d) Resize Image
Fig. 3. Preprocessing steps of Myanmar character

\section{A. Feature Extraction}

Selection of feature extraction methods is one of the key steps in achieving high recognition accuracy. After preprocessing, features for each character image are extracted based on Shape description. Shape description techniques can be generally classified into two classes of methods: contour-based methods and region-based methods. In this paper, region-based methods are used to extract features.The wide range of shape variations for handwritten characters requires an adequate representation of the discriminating features for classification. Eighteen features are extracted for the entire image based on the regional properties namely:

Area: It is calculated the actual number of pixels in the region.

Bounding Box: It is calculated the smallest rectangle containing the region.

Centroid: It is calculated the center of mass of the region. The first element of centroid is the horizontal coordinate of the centre of mass and the second element is the vertical coordinate.

Major Axis Length: It is calculated the length (in pixels) of the major axis of the ellipse that has the same normalized second central moments as the region.

Minor Axis Length: It is calculated the length (in pixels) of the minor axis of the ellipse that has the same normalized 
second central moments as the region.

Eccentricity: It is calculated as the ratio of the distance between the centre of the ellipse and its major axis length.

Euler Number: It is calculated as the difference of Number of Objects and Number of holes in the image.

Orientation: It is the angle between the $\mathrm{x}$-axis and the major axis of the ellipse that has the same second-moments as the region.

Extent: It is calculated as the ratio of pixels in the region to the pixels in the total bounding box.

Equiv Diameter: It is calculated the diameter of a circle with the same area as the region.

Solidity: It is calculated the proportion of the pixels in the convex hull that are also in the region.

Perimeter: It is calculated the distance around the boundary of the region.

Filled Area: It is calculated the number of pixels in filled Image.

Convex Area: It is calculated the number of pixels in convex image.

Table I shows the shape feature descriptors of Myanmar characters of $\mathrm{Ka}$, Kha, Ga, Gagyi and Nga.

TABLE I: SHAPE FEATURE DESCRIPTORS OF MYANMAR CHARACTER KA, KHA, GA, GAGYI, NGA

\begin{tabular}{|c|c|c|c|c|c|}
\hline $\begin{array}{l}\text { Characte } \\
\mathrm{r}\end{array}$ & $n$ & 2 & $\cap$ & 20 & $C$ \\
\hline Area & 125 & 72 & 72 & 79 & 69 \\
\hline Centroid & $\begin{array}{l}34.47,9.8 \\
4\end{array}$ & $\begin{array}{l}18.23,15 \\
79\end{array}$ & $\begin{array}{l}19.75,10 \\
19\end{array}$ & $\begin{array}{l}19.92,15 \\
94\end{array}$ & $\begin{array}{l}13.08,16 \\
01\end{array}$ \\
\hline $\begin{array}{l}\text { Boundin } \\
\text { g Box }\end{array}$ & $\begin{array}{l}0.5,0.5,71 \\
, 28\end{array}$ & $\begin{array}{l}0.5,0.5,29 \\
, 30\end{array}$ & $\begin{array}{l}0.5,0.5,37 \\
, 27\end{array}$ & $\begin{array}{l}0.5,0.5,46 \\
, 30\end{array}$ & $\begin{array}{l}0.5,0.5,32 \\
, 32\end{array}$ \\
\hline $\begin{array}{l}\text { Major } \\
\text { Axis }\end{array}$ & 95.99 & 40.76 & 56.21 & 61.10 & 52.08 \\
\hline $\begin{array}{l}\text { Minor } \\
\text { Axis }\end{array}$ & 31.19 & 31.90 & 32.04 & 24.31 & 34.93 \\
\hline $\begin{array}{l}\text { Eccentri } \\
\text { city }\end{array}$ & 0.94 & 0.622 & 0.821 & 0.917 & 0.741 \\
\hline $\begin{array}{l}\text { Orientati } \\
\text { on }\end{array}$ & 3.39 & 75.81 & 7.90 & -42.42 & 71.65 \\
\hline $\begin{array}{l}\text { Convex } \\
\text { Area }\end{array}$ & 1661 & 667 & 816 & 785 & 799 \\
\hline $\begin{array}{l}\text { Filled } \\
\text { Area }\end{array}$ & 133 & 73 & 74 & 79 & 69 \\
\hline $\begin{array}{l}\text { Euler } \\
\text { Number } \\
\text { Equiv }\end{array}$ & -1 & 0 & -1 & 1 & 1 \\
\hline $\begin{array}{l}\text { Diamete } \\
\mathrm{r}\end{array}$ & 12.61 & 9.57 & 9.57 & 10.02 & 9.37 \\
\hline Solidity & 0.075 & 0.107 & 0.088 & 0.100 & 0.086 \\
\hline Extent & 0.062 & 0.082 & 0.072 & 0.057 & 0.067 \\
\hline $\begin{array}{l}\text { Perimete } \\
\mathrm{r}\end{array}$ & 279.320 & 160.610 & 158.710 & 171.740 & 151.740 \\
\hline
\end{tabular}

Fig. 4(a) and 4(b) shows the shape features of centroid, bounding box, major axis and minor axis.

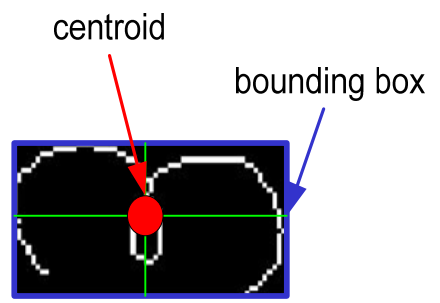

(a)Centroid and Bounding Box

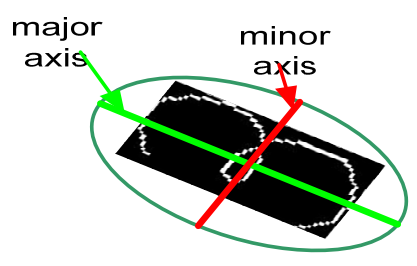

(b) Major Axis and Minor Axis

Fig. 4. Shape Features of Centroid, Bounding Box, Major Axis and Minor Axis

\section{Competitive Neural Trees (CNeT)}

The $\mathrm{CNeT}$ has a structured architecture. Each node contains slots and a counter age that is incremented each time a sample is presented to that node. The behavior of the node changes as the counter age increases. Each slot stores a prototype, a counter count, and a pointer to a node. The prototypes are updated to represent clusters of samples. The slot counter count is incremented each time the prototype of that slot is updated to match a sample.

Paper [6] proposed a structural adaptive intelligent tree (SAINT). The input feature space is hierarchically partitioned by using a tree-structured network that preserves a lattice topology at each sub network. Experimental results reveal that SAINT is very effective for the classification of a large set of real-world handwritten characters.

In paper [7], they proposed balanced neural tree to reduce tree size and improve classification with respect to classical neural tree. Two main innovations have been introduced (a) perceptron substitution and (b) pattern removal. The first innovation aims to balance the structure of the tree.If the last-trained perceptron largely misclassifies the given training set into a reduced number of classes, then this perceptron is substituted with a new perceptron.. The second novelty consists of the introduction of a new criterion for the removal of tough training patterns that generate the problem of over-fitting. The experimental results show that the proposed BNT leads to satisfactory results in terms of both tree depth reduction and classification accuracy.

Recently, a neural network tree (NNTree) classifier [8] using a multi layer perceptron (MLP) at each node was proposed for designing tree-structured pattern classifiers. To limit the depth of the tree, a new uniformity index was introduced. Such an index accomplishes the intuitive goal of reducing the misclassification rate. The performance of the NNTree has been evaluated in different contexts, such as in letter recognition satellite image classification and splicejunction and protein coding region identification. Experimental comparisons have been proposed with respect to other classifiers. The main drawback is the necessity of an ad hoc definition of some parameters for each context and training set, such as the network architecture (e.g., the number of hidden layers, number of nodes for each layer, etc.) and the uniformity index.

\section{A. CNeT Learning}

In the learning phase, the tree grows starting from a single node, the root. When a sample arrives at a node, all of its prototypes compete to match it. The distance measure used in this paper is the squared Euclidean norm, defined as 


$$
d\left(x, v_{j}\right)=\left\|x-v_{j}\right\|^{2}
$$

According to this scheme, the winner $V_{k}$ is the only prototype that is attracted by the input $X$ arriving at the node. The winner $V_{k}$ is updated according to the equation

$$
v_{k}^{\text {new }}=v_{k}^{\text {old }}+a\left(x-v_{k}^{\text {old }}\right)
$$

The learning rate $\boldsymbol{a}$ decreases exponentially with the age of a node according to the equation

$$
a=a_{0} \exp \left(-a_{d} a g e\right)
$$

where $a_{0}$ the initial value of the learning is rate and $a_{d}$ determines how fast $a$ decreases. The update (2) moves the winner $V_{k}$ closer to the sample $x$ and, therefore, decreases the distance between the two. After a sequence of sample presentations and updates, each of the prototypes will respond to samples from a particular sub region of the input space.

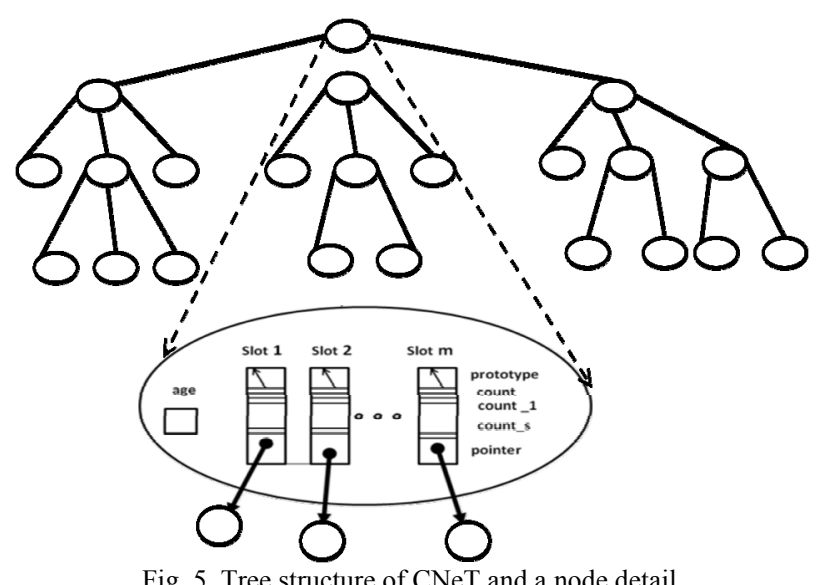

Fig. 5. Tree structure of $\mathrm{CNeT}$ and a node detail

\section{B. Life Cycle of CNeT}

Each node goes through a life cycle. The life cycle of a node may be partitioned into the following phases.

Creation: The node inherits properties from the parent slot such as the prototype and a fraction of the class counters.

Youth: The prototypes compete to respond to the samples and the winning prototype is updated. The prototypes split the region of the input space that the node sees into sub regions.

Maturity: The prototypes still compete for the samples and they are updated. If a splitting criterion is TRUE, then a new child is created and is assigned to a slot.

Frozen: the prototypes compete for the inputs but they are not updated. If the winner has a child-node assigned, then it sends the sample to the child.

Destruction: All children have been destroyed.

\section{Training Procedure of $\mathrm{CNeT}$}

Do while stopping criterion is FALSE:

1) Select randomly a sample $x$.

2) Traverse the tree starting from the root to find a terminal prototype $v_{k}$ that is close to $x$. Let $n_{l}$ and $s_{k}$ be the node and the slot that $v_{k}$ belongs to, respectively.

3 ) If the node $n_{l}$ is not frozen, then update the prototype $v_{k}$ according to (2).

4) If a splitting criterion for the slot $s_{k}$ is TRUE, then assign a new node as child to $s_{k}$ and freeze the node $n_{l}$.

5) Increment the counter count ${ }_{j}$ for class $C_{\boldsymbol{j}}$, the counter count in slot $s_{k}$, and the counter age in node $n_{l}$.

\section{Recall Procedures}

The objective of the recall procedure is to produce a class label $i=\ell(x), i \in \mathcal{L}$, for each input vector $x$. Presentation of samples in the recall phase begins at the root of the tree and proceeds down to the leaves. The prototypes belonging to internal slots are used as signposts for the search. More specifically, these prototypes guide the search algorithm to find a terminal prototype $v_{j}=v(x)$ that is close to the input vector without looking at all terminal prototypes. The same search method used during training is also called for recall with the input vector $x$ as the argument. The search will return a terminal prototype $v_{j}=v(x) \in V$, close to $x$.

\section{E. Global Search Method}

The global $(w)$ search method [9] expands the nodes of the tree level by level, starting at the root. After this is done for all the nodes that are to be expanded at this level of the tree, the prototypes with the smallest distances are selected. If a selected prototype has a child-node assigned, this childnode will be expanded during the next expansion step. Suppose a selected prototype is a terminal prototype. If its distance to the given feature vector is the smallest so far, then the smallest distance is updated and the prototype is the new candidate to be selected for return. When no more prototypes are to be expanded, the global $(w)$ search method terminates and returns the best terminal prototype seen.

\section{EXPERIMENTAL RESULTS}

In this paper, thirty three alphabet handwritten Myanmar characters are scanned with Canon Scan Lide 110 model .The training datasets are 660 and testing datasets are 330. The operation of a search method applied to a CNeT and trained to recognize handwritten characters using the feature vectors obtained after preprocessing stages. The $\mathrm{CNeT}$ was trained and grown using the global search method. Thirty three prototypes are selected and CNeT tree is constructed at learning rate $\alpha$ is 0.02 . Maturity age of the tree is 298 .

The root node of CNeT tree is randomly chosen from the training datasets and it has 33 slots for 33 handwritten Myanmar character prototypes. The Euclidean norm between current node and input sample node is calculated by using equation 1 and find the values of Euclidean norm between input sample node and features of all slots. The input sample node is created as a new node which is linked with that slot and current node and the prototype of that slot is copied to child node. The sample CNeT tree for Myanmar character recognition is illustrated in Fig. 7. The performance of the $\mathrm{CNeT}$ trained and grown using the global search method when the search width varied. As $w$ increases, the global method searches a larger subtree and is expected to return more often a terminal prototype that is the closest to the input example. Recognition accuracy rate 97\% is obtained with our testing data 330 . 


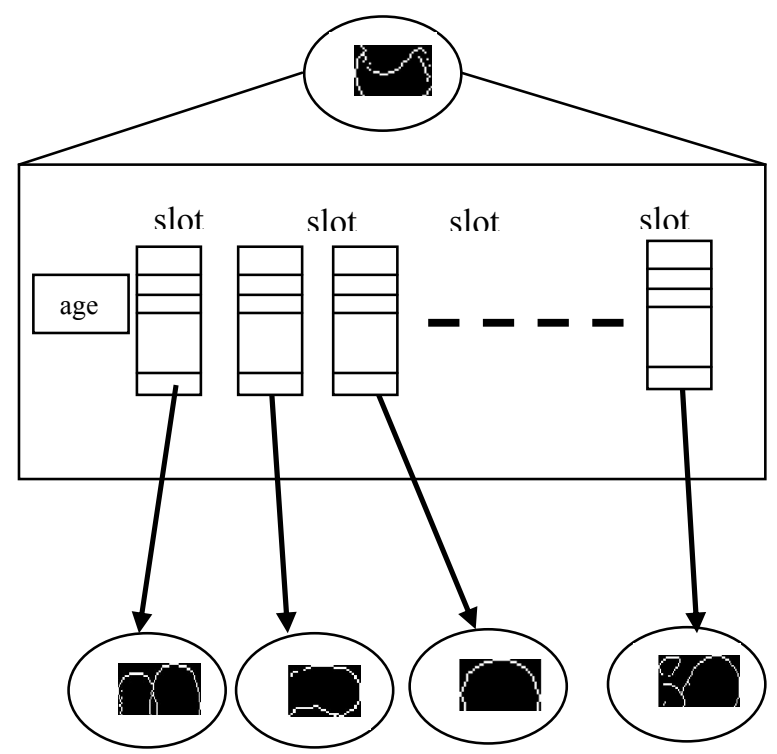

Fig. 6. Sample of Root Node for Myanmar Character CNeT Tree

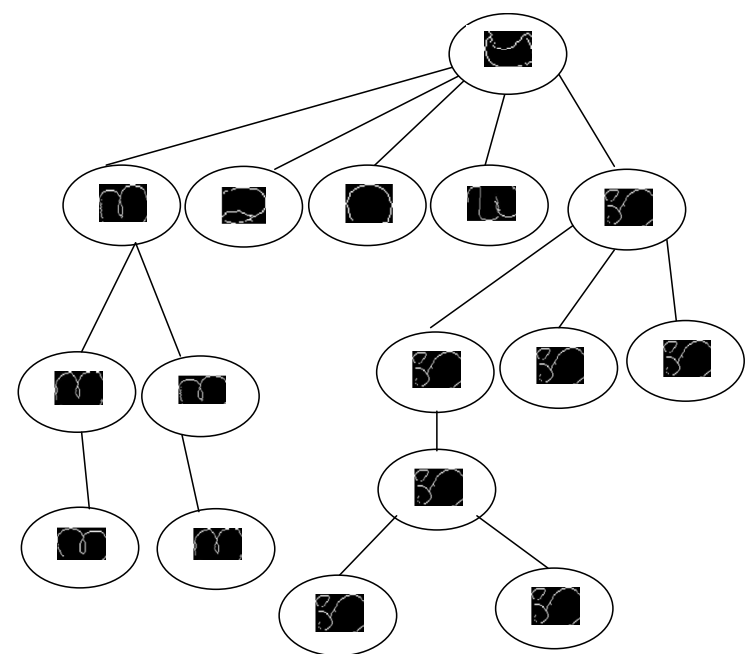

Fig. 7. Sample of CNeT Tree for Myanmar Character Recognition

\section{CONCLUSION}

This paper describes $\mathrm{CNeT}$ for character recognition. The performance of a trained $\mathrm{CNeT}$ depends on the search method employed in the learning phases. The global search method is presented in this paper. The $\mathrm{CNeT}$ grown using the global search method was allowed to reject some ambiguous samples in order to improve its recognition accuracy. The reliability and recognition accuracy of the trained $\mathrm{CNeT}$ can be improved by a recall strategy. The speed of the learning process is mainly determined by the computational complexity of the search method.

\section{REFERENCES}

[1] A. Bharath and S. Madhvanath, "Free Pad: a novel handwriting-based text input for pen and touch interfaces," in Proceedings of the $13^{\text {th }}$ international Conference on Intelligent User Interfaces, pp. 297-300, 2008.

[2] A. Bhardwaj, F. Farooq, H. Cao and V. Govindaraju," Topic based language models for OCR correction," in Proceedings of the Second Workshop on Analytics for Noisy Unstructured Text Data, pp. 107-112, 2008.

[3] K. Sandar, "Off-line Myanmar Handwriting Recognition using Hidden Markov Models," 2005.

[4] M. M. Nge, "Automatic Segmentation Of handwritten Myanmar Digits and Scripts on the Voucher Sheet," 2004.

[5] S. S. S.Yee and Y. Thein, "High Accuracy Myanmar Handwritten Character Recognition using Hybrid approach through MICR and Neural Network," IJCSI International Journal of Computer Science Issues, vol. 7, issue 6, November 2010.

[6] H. H. Song and S. W. Lee, "A self-organizing neural tree for large-set classification," IEEE Trans. Neural Networks, vol. 9, pp. 369-380, May 1998.

[7] C. Micheloni, A. Rani, S. Kumar and G. Foresti, "A balanced neural tree for pattern classification," NeuralNetworks, vol. 27, pp- 81-90, 2012.

[8] Maji, "Effeicient design of neural network tree using a single splitting criterion," Nero computing, vol.71, pp-787-800, 2008.

[9] S. Behnke and Nicolaos B. Karayiannis, "Competitive Neural Trees for Pattern Classification," IEEE Transactions on Neural Networks, vol .6, November 1998.

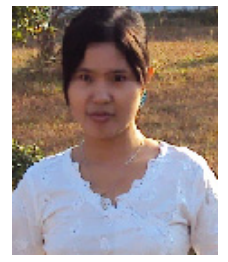

Theingi Htike received the master degree in computer science (M.C.Sc) from University of Computer Studies, Yangon (UCSY), Myanmar, in 2008. She is currently working toward the $\mathrm{PhD}$ degree in UCSY. Her research interests include image processing and optical character recognition.

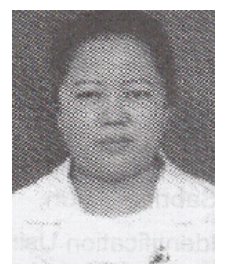

Yadana Thein received M.Sc (Master Computer Science) degree in 1996 and $\mathrm{PhD}$ (I.T) degree in 2007. She is now associated professor of U.C.S.Y (University of Computer Studies, Yangon). She has written about 25 papers altogether. About 10 of them are local papers and 15 are foreign papers. Her first paper is "Recognition of Myanmar Handwritten Digits and Characters "for ICCA conference in 2007. Her research interests include Image Processing, Neural Network and MICR (Myanmar Intelligent Character Recognition) field. 